\title{
March 2015 Critical Care Case of the Month: It's Not Always Sepsis
}

\author{
Dionne Morgan, MD \\ Carolyn H. Welsh, MD
}

University of Colorado and the Eastern Colorado Veterans Affairs Medical Center

Department of Medicine

Division of Pulmonary Sciences and Critical Care Medicine

Denver, CO

\section{History of Present IIIness}

A 57-year-old man with multiple co-morbidities including diabetes mellitus presented with wet gangrene of the right foot and hypotension. He had diabetic ketoacidosis and acute kidney injury. He was admitted to the medical intensive care unit, given intravenous fluids and treated with insulin therapy, piperacillin/tazobactam and vancomycin. Initial blood cultures grew Methicillin-resistant Staphylococcus aureus (MRSA). The podiatry service performed a right transmetatarsal amputation. Subsequently, he did well and was transferred to a medical floor for further care.

Three weeks later, following resolution of the initial sepsis, he developed persistently high fevers with hemodynamic instability despite continued antibiotic therapy. He was transferred back to the MICU for presumed sepsis.

\section{Past Medical History, Social History and Family History}

The past medical history was significant for diabetes, hypertension, COPD, coronary artery disease and hepatitis $\mathrm{C}$. He did not smoke nor drink alcohol. Family history was non-contributory.

\section{Physical Examination}

On readmission to the medical intensive care unit, the patient was noted to have a generalized maculopapular rash on both upper and lower extremities, torso, palms and soles of his feet, associated with facial and periorbital edema (Figure 1). There was no mucosal membrane involvement or lymphadenopathy. He was also febrile to $104^{\circ} \mathrm{F}$, hypotensive to $80 / 50 \mathrm{~mm} \mathrm{Hg}$ and icteric. 


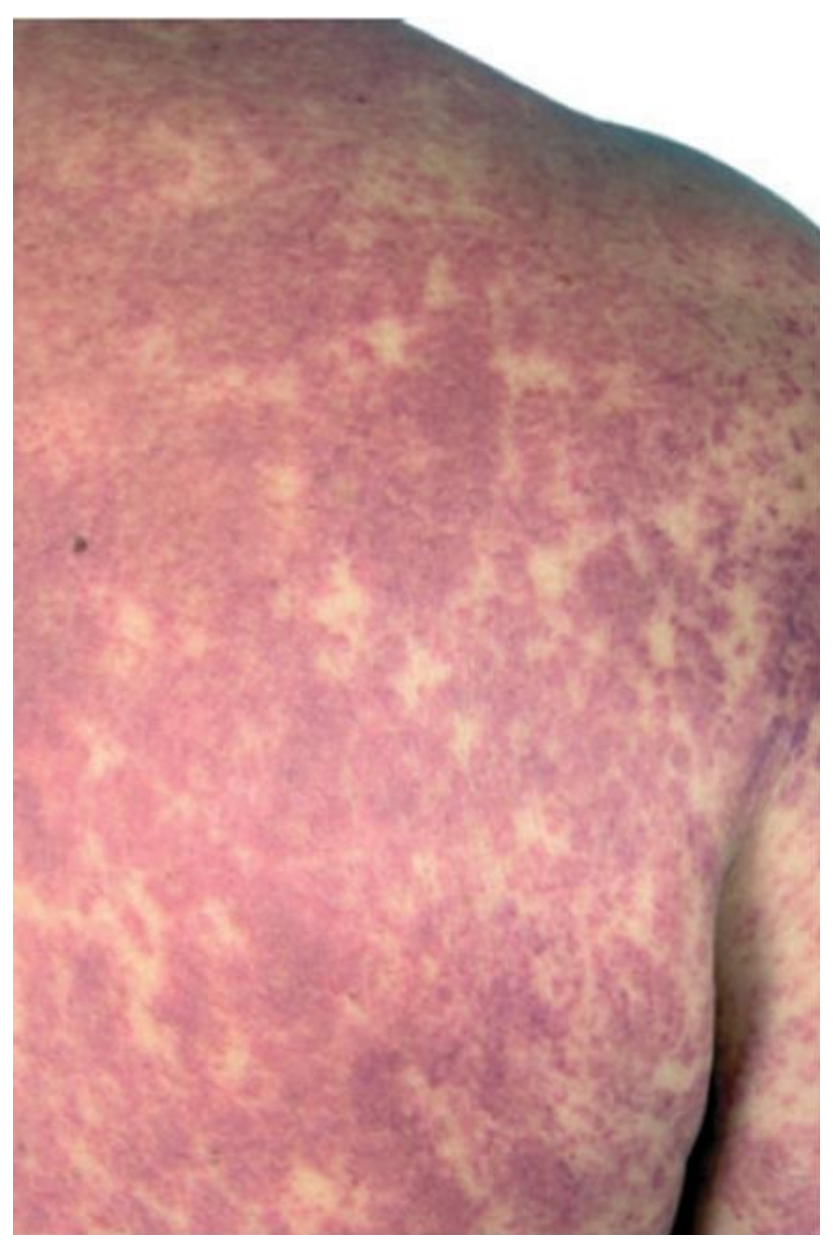

Figure 1. Image of rash.

\section{Laboratory Studies}

Initial labs showed elevated leukocyte count, BUN and creatinine with anion-gap metabolic acidosis but a normal liver enzyme profile. Repeat labs on readmission to the medical ICU were significant for severe leukocytosis, with marked eosinophilia, atypical lymphocytes on blood smear, acute transaminitis and hyperbilirubinemia.

Admission labs: White blood cell count (WBC) $29.9 \times 1000$ cells/ $\mu \mathrm{L}$. Eosinophils $0.0 \%$ (Normal 0.0 - 0.7\%), AST $28 \mathrm{U} / \mathrm{L}$, ALT $15 \mathrm{U} / \mathrm{L}$, ALP $162 \mathrm{U} / \mathrm{L}$, total bilirubin $0.2 \mathrm{mg} / \mathrm{dL}$. Labs on ICU readmission: White blood cell count (WBC) $35.7 \times 1000$ cells $/ \mu \mathrm{L}$.

Eosinophils 2.3\% (Normal 0.0 -0.7\%), AST 486 U/L, ALT 288 U/L, ALP 749 U/L, total bilirubin $4.3 \mathrm{mg} / \mathrm{dL}$.

Which are components of the SIRS criteria?

1. Elevated respiratory rate

2. Hypothermia

3. Leukocytosis

4. Tachycardia

5. All the above 


\section{Correct! \\ 5. All of the above}

The Systemic Inflammatory Response Syndrome (SIRS) is clinically defined as the presence of at least two or more of the following criteria: fever $>38.0^{\circ} \mathrm{C}$ or hypothermia $<36.0^{\circ} \mathrm{C}$, tachycardia $>90$ beats/minute, tachypnea $>20$ breaths/minute, leukocytosis $>12 \times 10^{9} /$ or leucopoenia $<4 \times 10^{9} /$. It is a manifestation of dysregulated inflammation and may be associated with both infectious processes and noninfectious processes such pneumonia, pyelonephritis, acute pancreatitis and burns. When SIRS is suspected it should prompt an evaluation for a septic infectious focus. Sepsis is a systemic inflammatory response to a confirmed or suspected infection. The development from SIRS to sepsis to septic shock represents a continuum with increasing mortality. Early treatment with broad-spectrum antibiotics and fluid resuscitation has been strongly related to increased survival.

\section{Hospital Course}

Despite broad-spectrum antibiotic coverage with vancomycin and piperacillin/tazobactam, the patient remained febrile although hemodynamically stable. Labs were notable for persistent severe leukocytosis, high eosinophil count, bandemia and rising liver enzymes. The erythematous rash progressed along his extremities and torso. Repeat blood and wound cultures were negative. Abdominal imaging including CT scanning of abdomen and pelvis showed no acute intrabdominal or hepatic pathology.

Which of the following diagnosis is/are a life-threatening skin condition?

1. DRESS syndrome

2. Pemphigus

3. Stevens Johnson Syndrome

4. Toxic Epidermal Necrolysis

5. Urticaria with angioedema

6. All the above 


\section{Correct! \\ 6. All of the above}

Drug reaction with eosinophilia and systemic symptoms (DRESS) syndrome is defined by the presence of at least three of the following findings: fever, exanthema, eosinophilia, atypical circulating lymphocytes, lymphadenopathy, and hepatitis. It is a severe drug hypersensitivity reaction with cutaneous, hematological and multi-organ involvement (1-4). Hemodynamic instability is seen and can be mistaken for septic shock. Estimated mortality rate is high at $10 \%$. Patients typically present with extensive mucocutaneous rash, accompanied by fever, lymphadenopathy, hepatitis, hematologic abnormalities with eosinophilia and atypical lymphocytes. Onset is typically 2-6 weeks after exposure to a medication. The most common visceral manifestation is acute hepatitis. Other organ system involvement can include myocarditis, colitis, encephalitis and rarely interstitial pneumonia with eosinophilia. Fulminant hepatic failure is the leading cause of death.

Stevens Johnson syndrome and toxic epidermal necrolysis are a spectrum of the same condition with toxic epidermal necrolysis affecting $30 \%$ of more of the skin and Stevens Johnson less than $10 \%$. These cause necrosis of the epidermis and sloughing of the mucous membranes and skin. Urticaria with angioedema is a pruritic raised area of the skin with swelling of dermis and subcutaneous tissue sometimes leading to anaphylaxis. Pemphigus is a bullous disease of autoimmune cause with autoantibodies to desmogleins. It can be associated with drug exposure, causes sloughing of the epidermis and may involve mucous membranes (5). These all can be life-threatening.

\section{Hospital Course (cont'd)}

A dermatology consult was obtained for this suspected drug reaction. The consensus was that the presence of persistent fever, and generalized rash with a systemic inflammatory response was likely due to a drug reaction known as Drug Reaction with Eosinophilia and Systemic Symptoms (DRESS syndrome). It was thought that the culprit drug was either piperacillin/tazobactam or vancomycin.

Exact pathogenesis of DRESS is unknown, but may involve failure of drug detoxification pathways or reactivation of human herpes viral infections. In some studies, HHV-6 reactivation has been suggested as a potential contributor with the virus detected in $41 \%$ of cases; and in one prospective study including 40 DRESS patients, Epstein-Barr virus, $\mathrm{HHV}-6$, and $\mathrm{HHV}-7$ reactivations were found in $76 \%$ of the cases. It has been shown that there is a drug-induced hypogammaglobulinemia that occurs in DRESS syndrome permitting viral reactivation of HHV-6, HHV-7, EBV, and CMV and this results in oligoclonal proliferation of activated virus-specific and nonspecific T cells (6). Eosinophilic accumulation is thought to account for the multi-organ involvement. 
What is the first step of recommended therapy for severe drug reactions such as DRESS syndrome?

1. Discontinue the culprit drug

2. High dose corticosteroids

3. IV immunoglobulin

4. Plasmapheresis 


\section{Correct! \\ 1. Discontinue the culprit drug}

Early recognition and prompt withdrawal of the culprit drug is essential. Numerous drugs have been implicated including anticonvulsants (e.g. phenytoin, carbamazepine), antidepressants (e.g. amitriptyline, fluoxetine), anti-inflammatory agents (e.g. naproxen, ibuprofen), anti-infectives (vancomycin, piperacillin/tazobactam, doxycycline), betablockers and angiotensin-converting enzyme inhibitors $(7,8)$.

Corticosteroids, given over a prolonged period of 6 to 8 weeks duration to avoid relapse, are the mainstay of therapy. There are no randomized controlled trials comparing supportive care alone to use of systemic corticosteroids however the eosinophilic disorders generally respond clinically to corticosteroid therapy. Other recommended therapeutic options include IV immunoglobulin and/or antiviral ganciclovir if symptoms progress despite corticosteroids (9).

Currently IV immunoglobulins plus high-dose steroids are considered second-line therapy. However, one recent observational study did not find any clinical benefit when IV immunoglobulin was used as a single treatment in DRESS (10). Initiation of second line therapy with IV immunoglobulins can achieve the desired response although the exact mechanism of the therapeutic effect in DRESS syndrome is not clear. There have been a few anecdotal case reports of the therapeutic benefits of plasmapheresis in DRESS syndrome.

Both antibiotics were consequently discontinued and as per recommendations, the patient was started on high dose prednisone with plan for slow taper over 6 weeks. Improvement in the patient's laboratory abnormalities with gradual resolution of the fever and rash was noted. By hospital day 38 , his leukocytosis and eosinophilia had completely resolved.

\section{References}

1. Cacoub P, Musette P, Descamps V, Meyer O, Speirs C, Finzi L, Roujeau JC. The DRESS syndrome: a literature review. Am J Med. 2011;124(7):588-97. [CrossRef] [PubMed]

2. Walsh SA, Creamer D. Drug reaction with eosinophilia and systemic symptoms (DRESS): a clinical update and review of current thinking. Clin Exp Dermatol. 2011;36(1):6-11. [CrossRef] [PubMed]

3. Fleming P, Marik PE. The DRESS syndrome: the great clinical mimicker. Pharmacotherapy. 2011;31(3):332. [CrossRef] [PubMed]

4. Criado PR, Criado RF, Avancini JM, Santi CG. Drug reaction with Eosinophilia and Systemic Symptoms (DRESS) / Drug-induced Hypersensitivity Syndrome (DIHS): a review of current concepts. An Bras Dermatol. 2012;87(3):435-49. [CrossRef] [PubMed] 
5. Samel AD, Chia-Yu C. Drug Eruptions. In: UpToDate Corona, R. (Ed). UpToDate. Waltham, MA. Available at: http://www.uptodate.com/contents/drug-eruptions (requires subscription, accessed on February 24, 2015).

6. Criado PR, Avancini J, Santi CG, Medrado AT, Rodrigues CE, de Carvalho JF. Drug reaction with eosinophilia and systemic symptoms (DRESS): a complex interaction of drugs, viruses and the immune system. Isr Med Assoc J. 2012;14(9):577-82. [PubMed]

7. O'Meara P, Borici-Mazi R, Morton AR, Ellis AK. DRESS with delayed onset acute interstitial nephritis and profound refractory eosinophilia secondary to Vancomycin. Allergy Asthma Clin Immunol. 2011;7:16. [CrossRef] [PubMed]

8. Jurado-Palomo J, Caba-as R, Prior N, Bobolea ID, Fiandor-Román AM, LópezSerrano MC, Quirce S, Bellón T. Use of the lymphocyte transformation test in the diagnosis of DRESS syndrome induced by ceftriaxone and piperacillin-tazobactam: two case reports. J Investig Allergol Clin Immunol. 2010;20(5):433-6. [PubMed]

9. Descamps V, Ben Saïd B, Sassolas B, et al. Management of drug reaction with eosinophilia and systemic symptoms (DRESS). Ann Dermatol Venereol. 2010;137(11):703-8. [CrossRef] [PubMed]

10. Joly P, Janela B, Tetart F, et al. Poor benefit/risk balance of intravenous immunoglobulins in DRESS. Arch Dermatol. 2012;148(4):543-4. [CrossRef] [PubMed] 